\title{
LES CERCLES CROISSANTS DE LA DÉSIDENTIFICATION PSYCHO- ET SOCIOGENÈSE DE LA HAINE RÉFLEXIONS SUR LE RWANDA
}

\author{
Abram DE SwaAn
}

Pourquoi des hommes manifestent-ils si souvent à l'égard d'autres hommes, des étrangers lointains qu'ils n'ont jamais rencontrés et ne rencontreront probablement jamais, des sentiments d'une rare intensité ? Il se peut que la forte charge affective de ces perceptions à distance soit un phénomène tout à fait récent, le complément émotionnel impliqué dans l'expérience individuelle de transformations sociales majeures.

\section{IDENTIFICATIONS ET DÉSIDENTIFICATIONS}

Dans un article qui fait pendant à celui-ci, j'ai soutenu que l'identification était un processus cognitif et émotionnel par lequel progressivement des hommes se perçoivent comme semblables aux autres ${ }^{1}$. Le contexte d'identification biogénétique et sociogénétique le plus ancien se fondait sur la parenté au sein des groupes de survie ${ }^{2}$. Ce n'est qu'avec l'extension de l'agriculture sédentaire qu'une seconde matrice sociogénétique d'identification émergea dans le contexte du village rural : la proximité. Pendant des millénaires, ces deux matrices, «sang » et «sol », furent les principaux vecteurs de l'identification. Aujourd'hui encore, elles nourrissent les métaphores mobilisatrices permettant d'évoquer des sentiments de ressemblance sur une échelle beaucoup plus grande: «enfants de Dieu», «frères d'armes », «patrie» ou «langue maternelle »et «aime ton prochain comme toi-même ».

1. SwaAn, 1995.

2. Freud, 1995, p. 46 : « Nous avons déjà deviné que l'une des tendances principales de la culture est d'agglomérer les hommes en de grandes unités. [...] Étant donné que l'être humain ne dispose pas de quantités illimitées d'énergie psychique, il lui faut venir à bout de ces tâches par une répartition appropriée de la libido. Ce qu'il consomme à des fins culturelles, c'est en grande partie aux femmes et à la vie sexuelle qu'il le retire. »

Revue de synthèse : $4^{\mathrm{e}} \mathrm{S} . \mathrm{n}^{\mathrm{o}}$ 1, janv.-mars 2001, p. 185-206. 
Jusqu'à l'époque moderne de la formation des nations, de l'émergence de la conscience de classe et des idéologies raciales, les identifications à grande échelle hors du cadre de la famille et du village demeurèrent assez rares. D'ores et déjà, des exceptions anciennes ont pu exister, telle la solidarité entre membres très dispersés de réseaux dynastiques et aristocratiques, ou entre des ordres monastiques disséminés, ou bien au sein des rangs de grandes armées. Dès le début, on exhortait les croyants à s'identifier dans la foi avec leurs lointains frères et sœurs, à l'instar des habitants du royaume qui étaient rappelés à leur devoir de loyauté envers leur souverain et ses nombreux sujets largement dispersés. Toutes ces identifications à grande distance s'exprimaient au travers de métaphores de parenté et de proximité, mais pour la plupart des gens du commun, leur portée émotionnelle n'était que secondaire et limitée.

L'identification est le complément émotionnel de la formation de groupe. Elle implique la réalisation au niveau affectif que les autres nous sont semblables, qu'ils appartiennent à notre propre groupe, et que d'autres en revanche sont différents, n'en font pas partie et doivent donc être exclus. Les tendances considérées comme négatives, de nature sexuelle, agressive, ou les penchants égoïstes niés en nous-même et chez nos pairs sont attribués à ces autres. Dans la théorie psychanalytique, cette combinaison de déni et d'attribution est appelée « projection ». Dans une interprétation plus élaborée, on considère que les caractéristiques projetées sont ensuite vécues par procuration par ceux qui les avaient antérieurement projetées sur l'autre, à l'intérieur d'un processus appelé «identification projective ». Le terme de «désidentification» employé dans le présent contexte fait référence à ce processus de déni, de projection et, selon le cas, d'expérience par procuration ${ }^{3}$.

C'est ce second processus qui sera examiné ici, processus d'exclusion sociale et de désidentification émotionnelle avec l'affect qui l'accompagne : la haine ${ }^{4}$. L'argumentation portera sur les événements qui, en 1994, ont conduit à un génocide au Rwanda. Dans la partie finale, le compte rendu sociopsychologique sera placé dans son contexte politique de

3. SwaAn, 1995. Pour une discussion des diverses significations de la notion d'« identification projective », voir Bateman et Holmes, 1994, ainsi que GabBard, 1994. Dans les écrits psychanalytiques, cette expression, forgée par Melanie Klein, fait souvent référence à la relation entre un patient et un thérapeute. On peut remarquer qu'elle dénote parfois le mécanisme par lequel l'analyste finit par s'identifier aux traits négatifs que le patient a projetés sur lui, dans le contexte du transfert et du contre-transfert. C'est la connotation interactive, relationnelle du concept, sa nature dialectique, qui nous intéresse dans le cadre « social » examiné ici.

4. FLETCHER, 1995, p. 289-291, mentionne explicitement « une contraction dans le champ de l'identification à l'autre » comme l'un des trois critères du «processus décivilisateur ». Voir aussi Elias, 1989, et Mennell, 1990. 
décomposition de l'État et de menaces militaires aux frontières extérieures.

L'identification et la désidentification ne sont pas en opposition. Elles se situent plutôt sur les deux côtés d'un triangle émotionnel dont la base est l'ignorance et l'indifférence. Dans leur vaste majorité, les êtres humains sont totalement étrangers les uns aux autres. On observe dans l'Histoire que la plupart des individus n'ont pas eu conscience de l'existence même de leurs semblables. Ce n'est qu'au cours de ces derniers siècles qu'un changement s'est produit, à un rythme accéléré. Pourtant, même si une conscience vague de la présence d'autres hommes sur des terres lointaines prévaut, l'indifférence règne, parfois accompagnée de fantasmes diffus et peu tenaces.

Ainsi, pour que des hommes éprouvent un sentiment quelconque à l'égard d'étrangers lointains, il leur faut d'abord découvrir leur existence, et si par la suite, ils en viennent à les haïr, ils doivent d'abord avoir été informés de leurs caractéristiques haïssables. Tout cela ne prêterait guère à conséquence s'il n'existait des interdépendances effectives de groupe à groupe. Les us et coutumes abjects des Ludimango, leur cannibalisme, leur diverses façons d'exploiter les femmes, de rôtir le gibier vivant, de martyriser les anguilles, les chats, les taureaux, de torturer les jeunes adolescents, d'exciser les jeunes filles, de brûler les veuves, rien de tout ceci ne sera bien excitant pour d'autres groupes aussi longtemps qu'ils n'ont pas affaire aux Ludimango.

C'est la transformation des relations sociales qui entraîne la transformation du sentiment, en un double mouvement d'identification et de désidentification qui remplace l'absence de conscience et l'indifférence antérieures. La plus grande portée de ces investissements émotionnels correspond à l'agrandissement du champ des relations sociales, c'est-à-dire aux relations de conquête, de conversion, d'échanges et de domination, mais surtout à l'extension du conflit. (Car le conflit est un jeu ambivalent qui implique déjà des intérêts opposés et des intentions semblables.)

L'esclavage transatlantique peut fournir un exemple de la transformation des relations et, en conséquence, des sentiments entre des peuples qui jusqu'alors s'étaient désintéressés quasi complètement l'un de l'autre. Lorsque les négriers européens et leurs complices africains procédaient à des rafles, pourchassaient, déportaient, vendaient et exploitaient les Africains par millions, ils n'étaient pas précisément en quête de matériau brut ou de fourrage. Ils étaient surtout à la recherche de travailleurs, de maind'œuvre humaine, d'êtres humains sensibles et compétents, capables d'exécuter des ordres, d'anticiper des récompenses et des punitions, de maîtriser la difficile technique de l'agriculture de plantation et le savoir-faire subtil du travail domestique. Autrement dit, il fallut que les marchands d'esclaves et les propriétaires s'identifient à leurs victimes en tant qu'êtres humains 
possédant des qualités semblables aux leurs, et que parallèlement ils se désidentifient d'eux en tant qu'êtres humains pourvus d'un registre de sentiments semblable, de valeur morale et d'une âme.

L'esclavage fut un fait concomitant de la naissance du système mondial moderne, c'est-à-dire de l'émergence du grand commerce triangulaire de l'Atlantique entre l'Europe, l'Afrique et les Amériques. Transformer les Africains en esclaves nécessita un travail socio-émotionnel considérable. Ils devaient être reconnus en tant qu'êtres humains et, en même temps, exclus comme «bétail » ou «singes » (malgré tout l'espèce la plus proche de l'homme), quasi animaux, sous-hommes. Dans le meilleur des cas, ils étaient considérés comme « enfantins », c'est-à-dire comme «pas encore » tout à fait humains, mais indéniablement sur la voie d'une pleine humanité sous la tutelle de gardiens sévères mais bienveillants. Le thème de l'esclavage n'est abordé que brièvement dans le présent contexte, afin de décrire les deux doubles mouvements en jeu dans la dynamique de l'élargissement du champ des investissements émotionnels : le champ agrandi des configurations sociales, c'est-à-dire l'émergence du commerce triangulaire de l'Atlantique, qui coïncide avec l'émergence d'un sentiment de portée élargie; et le double processus socio-émotionnel d'identification et de désidentification qui transcende l'inconscience et l'indifférence. D'une certaine façon, l'esclavage des plantations constitua « le péché originel de la modernité » : un vaste terrain d'expérimentation pour la déportation en masse, l'exploitation massive et le dénuement extrême dont la légitimation par des théories racistes ira croissant. Il fournira le premier exemple de travail socio-émotionnel d'identification et de désidentification à l'échelle transcontinentale.

\section{L'EXTENSION DU CHAMP DE L'AUTORITÉ POLITIQUE}

À l'origine, l'opinion occidentale perçut les massacres au Rwanda comme un déchaînement spontané et catastrophique de "haine tribale » couvant de longue date. Aujourd'hui, de nombreux documents probants établissent que l'extermination massive des citoyens rwandais fut le point culminant d'une campagne bureaucratique soigneusement préparée et orchestrée, utilisant des moyens de communication de masse modernes, la propagande, l'administration publique et la logistique militaire. La catégorisation des habitants du Rwanda (et du Burundi) en «Tutsi » et « Hutu »a subi une série de changements au cours du siècle dernier. Elle a fini par recouvrir des significations multiples très difficiles à distinguer, variables dans le temps et d'une communauté à l'autre. Le contexte social embrassait 
des transformations politiques et économiques dans le sens d'une interdépendance à plus grande échelle.

En effet, il est exact que, longtemps avant la conquête européenne des terres du Rwanda et du Burundi actuels par les envahisseurs allemands, des systèmes politiques relativement stables et complexes étaient en place dans la région des Grands Lacs. Ainsi des « royaumes » étaient établis exerçant une forme d'autorité sur de vastes parties de la région ${ }^{5}$. Cependant, lesdits royaumes centraux avaient peu d'impact sur la vie quotidienne dans les villages, et leur emprise sur l'état d'esprit de leurs sujets était moindre. Lucien Bäck ${ }^{6}$ conclut.

«La plupart des auteurs les plus anciens représentaient le Rwanda comme un " royaume sacré" ou comme une "monarchie absolue". À la lumière de la recherche empirique la plus récente, il apparaît que le rôle de l'umwaami et de la cour qui lui était directement rattachée fut probablement plus limité, y compris au cours des dix-neuvième et vingtième siècles lorsque la monarchie parvint à étendre son influence de manière considérable [...]»

Trouwborst remarque : «Pour ces différentes raisons, il est difficile de soutenir sans réserves que l'économie politique des États interlacustres eut un caractère centralisé pouvant être considéré comme un tout administré centralement ${ }^{7}$.» Puis, il poursuit : « J'en induis donc que concernant les dépenses également, la redistribution eut une dimension très décentralisée. Il est exact que le roi était au centre de l'État, mais il n'occupait cette position qu'en un sens fondamentalement symbolique ${ }^{8}$. » Et Trouwborst conclut, non sans réserves : «Une économie politique, au sens d'une administration publique au service des intérêts de l'État et de ses sujets, était pratiquement inexistante ${ }^{9}$. »

Il est clair qu'au cours des derniers cent cinquante ans, la région des Grands Lacs a subi une transformation sociale majeure, fortement accélérée au cours des périodes coloniales et postcoloniales. Elle est passée de politiques et d'économies essentiellement segmentées et décentralisées aux États et aux marchés beaucoup plus centralisés de l'Ouganda, du Rwanda et du Burundi contemporains. Cependant, ce processus, loin d'être exceptionnel au sein du continent africain, était le processus normal de formation des États et de développement économique dans la région ${ }^{10}$. Ainsi, l'agran-

5. Sagan, 1985, p. 3-58; Newbury, 1988; Prunier, 1997; et Lemarchand, 1996. Sur l'histoire politique de la conquête coloniale du continent africain, voir WesseLing, 1991, p. 169205.

6. Вёск, 1981, p. 30 .

7. Trouwborst, 1991, p. 99.

8. Trouwborst, 1991, p. 102.

9. Trouwborst, 1991, p. 105.

10. BAyART, 1989. 
dissement du champ de l'organisation sociale au Rwanda ne suffit pas à expliquer les événements extraordinaires (même s'ils ne sont pas uniques) qui s'y sont déroulés. Il n'en constitue qu'une condition nécessaire.

Dans la présente argumentation, cette transformation sociale forme le contexte d'une transformation des investissements émotionnels ${ }^{11}$. Il s'agit maintenant d'examiner les glissements de sens intervenus dans l'opposition majeure qui a conduit à l'affrontement entre Rwandais : le conflit entre « Tutsi » et « Hutu ». On ne peut donner ici qu'une analyse assez schématique de relations sociales en évolution et entrecroisées d'une complexité qui reste déconcertante ${ }^{12}$.

\section{LE REGARD DES AUTRES. VICISSITUDES DE L'OPINION DES SPÉCIALISTES}

Les spécialistes contemporains sont unanimes à rejeter les premières interprétations de la paire conceptuelle «Tutsi »/«Hutu» comme relevant de catégories raciales duelles et fixes. Mais paradoxalement, c'est, ironie tragique d'un genre connu ${ }^{13}$, ce que ces termes ont fini par suggérer dans le discours politique actuel, influencé par des écrits scientifiques plus anciens.

Les premiers auteurs missionnaires et ethnographes choisissaient leurs informateurs presque exclusivement parmi les aristocrates de la cour. Ces derniers s'identifiaient comme «Tutsi », et suggéraient que leurs pairs avaient toujours détenu le pouvoir en tant que groupe dirigeant héréditaire. Sans doute s'agissait-il d'un genre de fiction ex post que les oligarchies en place ont coutume de créer (et de tenir pour vraie après une ou deux générations). Les premiers anthropologues allemands attribuèrent à cette classe dirigeante certains caractères physiques, un «type normatif somatique ${ }^{14}$ »: haute stature, front haut, mains et pieds étroits et allongés, nez fin et long, par comparaison aux «Bantu» de la classe paysanne «Hutu», trapus, au nez épaté et aux lèvres épaisses. À l'époque, les membres du groupe dirigeant s'identifièrent volontiers à l'image d'eux-mêmes que leur renvoyaient les spécialistes étrangers. Aujourd'hui, les «Tutsi » rejètent la division binaire et héréditaire entre «Tutsi » et « Hutu», lui préférant des catégories politiques et socio-économiques, mais peut-être s'agit-il là d'une

11. Newbury, 1988, p. 18 : «Ces transformations et d'autres, liées au développement du pouvoir politique et à l'expansion du capitalisme, ont été cruciales pour le développement d'une conscience politique au sein de la population rurale, et dans la configuration et l'émergence politique de l'ethnicité au Rwanda. »

12. Voir, cependant, Newbury, 1988, et MaLkKi, 1995.

13. Thomas et Thomas, 1928, p. 572 : «Si les hommes définissent des situations comme réelles, elles sont réelles dans leurs conséquences. »

14. Hoetink, 1962. 
«posture publique ${ }^{15}$ ». Et c'était leur déni même de différences fondées au nature entre les deux groupes qui était particulièrement insupportable aux idéologues du Hutu power.

Ces images rappellent les théories raciales appliquées à l'aristocratie française, dont on affirmait qu'elle était de souche «germanique » : taille élevée, composée de blonds aux yeux bleus, par contraste avec les Français du menu peuple présentés comme basanés, petits, trapus et d'origine celtique, selon les théoriciens racistes de l'époque dont le plus important était Joseph Gobineau ${ }^{16}$. Les anthropologues allemands, quant à eux, observaient que les premiers habitants des royaumes de la région interlacustre (Ouganda, Rwanda et Burundi) étaient d'origine bantu ou «négroïde », alors que les «Tutsi » étaient assimilés à la souche «éthiopienne» ou «nilotique », et descendaient des « Hamites » ou des « Sémites » ${ }^{17}$.

Les Belges reprirent le point de vue des spécialistes allemands et lui donnèrent une traduction administrative. Conformément à leur politique d' « autonomie locale », ils favorisèrent des officiels autochtones aux postes d'administrateurs locaux, nombre d'entre eux étant recrutés dans l'aristocratie de cour identifiée avec les «Tutsi » (même si une grande partie d'entre eux étaient considérés comme d'origine « hutu »). À partir de 1926, ils mirent en place un bureau d'état civil municipal comprenant un fichier de tous les citoyens avec leur photographie et la mention de leur appartenance ethnique ${ }^{18}$.

Les spécialistes contemporains ont totalement réfuté les catégorisations de leurs prédécesseurs. En premier lieu, aucune preuve ne vient aujourd'hui corroborer la thèse d'une invasion des «Tutsi » pastoraux et de leur installation au sein des peuples bantu de la région des Grand Lacs. En second lieu, il s'avère que les pratiques linguistiques, religieuses et culturelles en vigueur sont quasi identiques, et que la distribution des caractères physiques ne correspond en rien aux catégories définies. En conséquence, les spécialistes tendent généralement à penser que pendant plusieurs siècles, «Tutsi » et «Hutu» ont dû appartenir à la même entité culturelle et endogamique, et qu'ils pourraient bien faire partie du même groupement génétique $^{19}$.

15. Pour une discussion du débat sur «l'ethnicité », et en particulier sur le déni de toute différence ethnique au sein de la classe dirigeante burundaise, «l'amnésie ethnique comme choix rationnel », voir LEMARCHAND, 1996, p. 30-33.

16. Gobineau, 1853.

17. Voir Prunier, 1997, p. 14-21, pour l'examen des premières interprétations parfois très déconcertantes des origines des «Tutsi ».

18. À ce jour, je n'ai trouvé aucune trace des règlements administratifs relatifs à l'attribution ethnique.

19. Cette position a fait l'objet d'une déclaration à l'emporte-pièce dans Le Monde du 12 novembre 1996, p. 10 : «Dominique Franche, géographe » : «Il n'y a qu'une seule ethnie au Rwanda, l'ethnie rwandaise. » 
Bäck $^{20}$ a effectué un résumé des différents points de vue des experts actuels sur les Rwandais qui mérite une ample citation :

«La population se répartit généralement en trois groupes distincts : Hutu (qui représentait $82,7 \%$ de la population totale en 1956), Tutsi $(16,6 \%)$ et Twa $(0,7 \%)$ [...] Sa distribution géographique est inégale. Dans le nord et le nordouest du pays, la majorité écrasante est hutu. Ailleurs, les Tutsi et la plupart des Twa sont disséminés parmi les Hutu. En dépit de particularités relatives aux régions et aux groupes, on peut dire que l'ensemble des Rwandais partagent une culture commune. Ceci est confirmé notamment par le fait que tous parlent la même langue avec des variations locales mineures, l'ikinyarwanda, très proche de l'ikirundi, idiome parlé dans le Burundi voisin. On a soutenu que les trois groupes différaient par des caractères physiques distincts. On décrit généralement les Hutu comme «Bantu», les Tutsi comme «Éthiopiens » et les Twa comme «Pygmées » [...] Cependant, les données anthropométriques ne corroborent pas systématiquement cette description [...] En conséquence, la division en Hutu, Tutsi et Twa semble essentiellement correspondre à une réalité d'ordre social ${ }^{21}$. »

Bäck ${ }^{22}$ conclut ainsi son résumé des découvertes récentes sur l'histoire de la population rwandaise : «Le fait que Hutu et Tutsi parlent la même langue et partagent une culture commune suggère qu'ils ont certainement vécu ensemble pendant une période bien plus étendue que simplement quelques siècles, voire qu'ils sont en fait de la même souche. » Et jusqu'en 1988, dans le Rwanda rural, le fossé ethnique ne constituait en aucun cas le seul et universel clivage. Les paysans se permettaient d' " inoffensives plaisanteries » ethniques même en « compagnie mixte », la politique du village visant à dresser les locaux contre les officiels de l'extérieur, plutôt qu'une ethnie contre l'autre. Mais cette situation allait bientôt changer sous l'impact des conflagrations ethniques dans le Burundi voisin et de la propagande dans les médias rwandais ${ }^{23}$.

UNE PARENTHÈSE SUR L'IDENTIFICATION DES AUTRES

Toute discussion portant sur les différences extérieures entre catégories sociales est — à juste titre — jugée déplacée au sein de la bonne société.

20. Вёск, 1981, p. 17.

21. Lemarchand, 1996, p. 1-16, étaye solidement le même argument. Les références de Bäck ne sont pas mentionnées dans la citation.

22. Вӓск, 1981, p. 18.

23. Lame, 1966, p. 73, sur le village de Murundi. 
Mais nous ne sommes pas ici dans le cadre d'un discours bienséant. L'identification émotionnelle avec les autres et la désidentification affective d'avec les autres présupposent d'abord l'identification des autres. Les catégories affectives et morales requièrent une construction cognitive, ou pseudo-cognitive, préalable. Jusqu'à présent, au Rwanda, cela se réalisait dans le cadre de l'interaction quotidienne en fonction de la réputation, de l'expérience, de l'intuition et des impressions. À partir des années vingt, on compléta cette pratique informelle par des techniques administratives d'identification : registres d'état civil, papiers d'identité, passeports, photos et ainsi de suite. Pourtant, ces mesures ne mirent pas fin au débat public où se posait l'éternelle question de savoir qui était «Tutsi », qui était «Hutu », et comment les différencier les uns des autres. En revanche, ce sont précisément les partisans les plus fanatiques d'une division héréditaire entre «Tutsi » et «Hutu » qui restent obsédés par la possibilité de « Tutsi » se faisant passer pour «Hutu », afin de plonger ces derniers dans la confusion et de les abuser. Il semble qu'aujourd'hui encore, il soit très difficile de se prononcer à partir des seuls critères physiques, même pour ceux dont la vocation est de faire valoir la distinction. Ceux-ci s'en remettent plutôt aux cartes d'identité établies à partir du registre d'état civil, dont ils déplorent aussi la falsification depuis toujours.

Or, toutes les divisions qui produisent un effet social, y compris celles qui sont étroitement liées à des distinctions somatiques aisément observables, et qui peuvent fort bien être fondées sur des différences génétiques, sont « essentiellement d'ordre social ». D'autre part, des particularités physiques évidentes « héritées » d'une génération à l'autre peuvent exister sans nécessairement avoir de fondement génétique : une haute taille est une bonne illustration parce qu'elle est en étroite corrélation avec une alimentation de qualité supérieure. Jusqu'à très récemment, et en de nombreuses régions du monde, une alimentation adéquate était le privilège des nantis qui transmettaient leur fortune et leur taille à leur descendance. Un teint clair, un regard ferme, un maintien droit, une voix sonore, une démarche vigoureuse, bref, tout un habitus qui semble caractériser l'apparence physique générale, et notamment marquer le contraste entre les apparences propres aux puissants et aux pauvres, peut être transmis des parents aux enfants, et cependant manquer de tout fondement génétique ${ }^{24}$.

Ainsi, des différences héritées significatives portant sur l'aspect physique peuvent exister d'un groupe social à l'autre. Elles n'ont pas leur origine dans des souches génétiques distinctes, mais s'inscrivent à l'intérieur de différences socialement héritées liées à la richesse, au prestige et au

24. Les riches n'ont pas seulement plus d'argent que les pauvres, ils sont aussi plus beaux. Ils peuvent plus aisément sélectionner des partenaires sains et bien formés, grands, sans déficiences ou tares sociales. Ils sont donc en mesure de reproduire un génotype social désirable. 
pouvoir. C'est par le processus social de la sélection sexuelle, c'est-à-dire par l'accouplement sélectif, que ces différences sociales peuvent en fin de compte constituer la cause de divergences génétiques entre les différents groupes endogamiques ${ }^{25}$. Finalement, un type normatif somatique unique peut très bien être absent. Néanmoins, le groupe social sera toujours reconnaissable en raison d'une « ressemblance familiale » (ce que Sudwig Wittgenstein a apellé «family likeness »), au sens à la fois littéral et wittgensteinien $^{26}$. Toute une série de types somatiques réciproquement très différents peuvent même exister côte à côte, considérés chacun comme caractéristique du groupe, figurant chacun un nœud à l'intérieur du réseau des ressemblances familiales, et dont l'origine peut se situer au sein d'un réseau d'intermariages spécifiques. Ce dernier cas paraît s'appliquer aux Juifs européens : ils ne se ressemblent absolument pas, et cependant certains frappent l'observateur intéressé comme «très juifs », c'est-à-dire comme présentant une ressemblance familiale avec l'un des quelque douze «types juifs » qu'il a mémorisé sous la forme d'une Gestalt. Ainsi, le cas peut fort bien se présenter de «Tutsi » au type très « tutsi », ce qui signifie également différent d'un «Hutu », sans impliquer qu'ils soient issus d'une souche génétique différente. Plusieurs types «tutsi » très distincts pourraient exister. Chaque type «tutsi » serait l'aboutissement de différences socialement héritées dues à l'alimentation et à la socialisation, renforcées par un intermariage sélectif, avec pour résultat final une différenciation génétique observable, mais mineure. Cela expliquerait que les Rwandais peuvent parfois identifier avec justesse les «Tutsi », et donc également les «Hutu », mais que souvent ils ne peuvent trancher, ou qu'ils font des assimilations erronées. Ce serait également l'explication d'un paradoxe majeur de la propagande du Hutu power : d'une part, elle soutient que les «Tutsi » ont une apparence très différente de celle des «Hutu» en raison de leur héritage génétique, mais, d'autre part, elle accus les «Tutsi » de tenter continuellement de se faire passer pour «Hutu» dans un but malfaisant, et d'y parvenir fort bien. Parce qu'il s'agit d'un thème si central du travail socio-émotionnel de la propagande « hutu », il paraît nécessaire d'adopter une position agnostique et soigneusement argumentée sur la question. Sa traduction typographique est rendue ici par le recours systématique aux guillemets encadrant les termes de la paire conceptuelle « Tutsi »/« Hutu».

25. Sur l'accélération de l'évolution génétique par la sélection sexuelle, voir DAwKINS, 1986, p. 199-216.

26. BLOK, 1975. 
« HUTU » ET « TUTSI ». DES LIENS PERSONNELS AUX STÉRÉOTYPES GÉNÉRAUX

Mais à quoi ces termes se référaient-ils initialement, avant d'être absorbés par le discours de l'anthropologie européenne, puis réintroduits dans le vocabulaire politique interlacustre? Ces premières connotations sont très difficiles à reconstruire étant donné la rareté des sources écrites précoloniales qui pourraient nous renseigner sur les significations anciennes des termes. Quelques informateurs, témoins de la colonisation allemande, se souviennent encore de ces premiers sens. Mais ils ont involontairement assimilé les connotations ultérieures venues s'ajouter à l'époque de la domination coloniale belge, et postcoloniale. Cependant, des indices permettent d'éclairer les multiples significations persistantes de la paire conceptuelle. Reportons-nous à Liisa Malkki et à son excellent compte rendu de recherche au sein des réfugiés « hutu » en Tanzanie, en $1986^{27}$, ainsi qu'aux minutieuses interviews de Catharine Newbury auprès d'habitants anciens de la région de Kinyaga ${ }^{28}$.

Sans rendre pleinement justice au compte rendu nuancé de Malkki, nous pouvons repérer trois niveaux de signification au moins. Le premier admet comme évident l'existence de catégories « tutsi » et « hutu » héréditaires et distinctes :

«Dans la construction mythico-historique, il est clair que les Tutsi font d'abord figure d'étrangers - historiquement venus "du Nord", " de Somalie ", ou " du Nil " à une époque récente. À cet égard, on a souvent affirmé que les Tutsi étaient d'origine "hamite", étrangers à la terre des Hutu "Bantu" ${ }^{29}$."

Selon ce mythe, les envahisseurs « tutsi » conquirent des terres qui autrefois avaient appartenu aux «Hutu ». Mais cette appropriation ne s'effectua pas par le seul recours aux armes. Et c'est ici que la paire «Tutsi »/ «Hutu » prend un second niveau de signification; eux, les «Tutsi », firent preuve de ruse : «[...] les Tutsi, qui possédaient le “don inné” de l'art de ruser, abusèrent et asservirent les premiers habitants du Burundi en leur faisant don de vaches ${ }^{30}$. »

27. Malkki, 1995. Cependant, voir également Gourevitch, 1996, pour un compte rendu très critique de l'ouvrage de Malkki et la réponse de MaLkKi, 1996.

28. Newbury, 1988.

29. MaLKKI, 1995, p. 68.

30. MALKKI, 1995, p. 68. 
Dans ce contexte, le terme fait très approximativement référence à un rapport de clientèle ou de servage endetté, entre un pasteur propriétaire de bétail et un agriculteur. Le premier prête quelques vaches au second qui lui verse une dîme sous forme de produits et est soumis à des corvées. Le rapport semble pris dans un réseau politiquement hiérarchisé qui transforme l'échange économique en un lien de sujétion à long terme :

« [...] avant l'arrivée des Tutsi, les Hutu n'étaient nullement Hutu : ils étaient simplement abantu [...] ce qui, en Kirundi, signifie "les peuples bantu ", ou simplement, "êtres humains. " [...] Les réfugiés affirmaient que la dénomination Hutu avait été introduite par les Tutsi originaires du Nord. Hutu signifie "esclave" ou "serviteur". Ainsi [...] " nous sommes devenus leurs esclaves" ${ }^{31}$. »

C'est ce que les «Hutu » burundais de Tanzanie interrogés par Malkki pensaient.

Un très grand nombre de preuves historiques corroborent la prédominance de telles relations d'échange inégales et de déférence, imposées par l'institution politique et l'ascendance militaire dans le Rwanda précolonial et colonial. Newbury fait remarquer que dans le sud-ouest du Rwanda, à Kinyaga, les rapports de clientèle imposèrent d'entrée des obligations collectives à la lignée, mais que progressivement ces liens umuheto firent place à des relations de dépendance ubuhake, impliquant des obligations et des droits individuels. Sous le régime colonial, ces liens de subordination furent progressivement transformés en outils d'oppression et d'exploitation $^{32}$.

En dehors de ses connotations « ethniques » ou «culturelles », du moins au Burundi, le terme «Hutu» possédait et recouvre toujours une signification supplémentaire :

«Dans son second sens, Hutu désigne un "subalterne " par rapport à un supérieur hiérarchique [...] "fils social " serait probablement même plus adéquat, puisque l'expression dénote non seulement une infériorité sociale, mais aussi un certain degré d'affectivité [...] Ainsi un Tutsi se présentant comme client vis-à-vis d'un patron plus riche sera désigné comme "Hutu", même si son identité culturelle reste tutsi ${ }^{33}$.»

À ce niveau, la paire «Tutsi »/« Hutu » dénote une relation spécifique, politiquement et économiquement contextualisée, qui peut se comparer à

31. MALKKI, 1995, p. 71.

32. NewBury, 1988, p. 115-140.

33. Lemarchand, 1996, p. 10. Dans ce contexte, la signification de l'expression «identité culturelle » est peu claire. 
celle, disons, d'un propriétaire vis-à-vis de son locataire, d'un créditeur visà-vis de son débiteur, ou d'un maître vis-à-vis de son serviteur. L'échange avait lieu entre un pasteur et un paysan. Il impliquait la faculté de disposer de vaches contre l'exécution de travaux.

La notion de relation précise et locale entre deux personnes face à face, même inégale et mal acceptée, se transforma ensuite en un concept généralisé et décontextualisé de relation entre deux catégories intemporelles, irréconciliables et hostiles ${ }^{34}$. Cela fut accompli par l'introduction de la construction mythico-historique de la conquête des «étrangers tutsi » et de leur asservissement des «indigènes hutu». Alors qu'il se référait à un passé lointain, ce niveau de signification fut utilisé pour faire passer le second niveau de signification, qui se référait à un passé beaucoup plus récent et toujours inscrit dans les mémoires, à un troisième niveau de signification dont le but était de fournir une nouvelle interprétation des événements vécus : à ce dernier stade d'interprétation, tous les ancêtres «tutsi » avaient été des conquérants exploitant tous leurs prédécesseurs « hutu». Des « Tutsi » qui se seraient comportés différemment n'auraient pu être de vrais « Tutsi ». Tous les « Tutsi » actuels cherchent à rétablir leur hégémonie politique et économique quels que soient les moyens à mettre en œuvre, et lorsqu'ils ne le font pas, c'est qu'ils ne sont pas de vrais « Tutsi ». Il faut qu'il en soit ainsi, indépendamment des relations antérieures entre « Tutsi » et «Hutu», indépendamment même de l'invasion et de l'oppression anciennes. C'est ce qui est fatalement inscrit dans l'essence «tutsi ${ }^{35} »$. À cet ultime niveau de signification, tous les «Tutsi » partagent des traits communs, qu'ils s'appliquent à leur apparence extérieure ou à leur psychisme. Leur trait dominant est leur appétit de pouvoir, moteur de toutes leurs entreprises. Pourtant, et c'est à remarquer, toujours selon la même idéologie du Hutu power, les «Tutsi » possèdent des traits qui leur assurent une suprématie sur les «Hutu»: les «Tutsi » sont plus intelligents; ils sont plus loyaux entre eux; et les femmes «tutsi » sont plus belles. Toutefois, les «Tutsi » mettent ces qualités au service de leur soif de pouvoir exclusivement. Par conséquent, les «Tutsi », retors et rusés ${ }^{36}$, n'utilisent leur intelligence que pour duper les «Hutu». C'est moins par le pur exercice du pouvoir que par stratagème qu'ils ont réussi à soumettre les

34. Pour une discussion du statut de la distinction entre relations affectives «proches » et «distantes » dans l'histoire de la théorie des sciences sociales, voir CALHoun, 1991, et les commentaires pertinents de SHILs, 1991.

35. MalkKi, 1995, p. 66 : «[...] dans la construction mythico-historique, la différenciation oppositionnelle essentielle — celle entre les Hutu et les Tutsi — fut élaborée à plusieurs niveaux : culturel, social, politique et physique. Chaque niveau renvoyait aux autres et leur combinaison établissait une différence catégorielle fondamentale et irréconciliable entre Hutu et Tutsi » (souligné par l'auteur).

36. La question se complique d'autant plus du fait de l'admiration des réfugiés « Hutu » pour les vols et stratagèmes réalisés par leur propre peuple, voir MALKKI, 1995, p. 186. 
«Hutu» et à s'approprier leurs terres ${ }^{37}$. Les « Hutu » se décrivent comme un peuple « simple», «frugal » et « honnête», mais aussi comme «primitif », «naïf » et «non civilisé » ${ }^{38}$. Les « Tutsi » n'utilisent leur loyauté réciproque qu'en fonction de leur lutte ouverte et clandestine pour le pouvoir. De même, ils exploitent la beauté de leurs femmes pour pousser au mariage les hommes « hutu », de sorte que tôt ou tard les épouses pourront être contraintes à espionner et à trahir leur mari ${ }^{39}$.

\section{LA MOBILISATION DU FANTASME}

Il n'est pas impossible que les « Hutu » burundais interrogés par Malkki au milieu des années quatre-vingt dans les camps de réfugiés tanzaniens aient été des idéologues «naïfs », voire dilettantes. Mais, dans le Rwanda du début des années quatre-vingt, leurs thèses étaient reprises presque textuellement par les professionnels du journalisme travaillant pour la revue du Hutu power, Kangura, et leurs confrères de Radio Mille Collines. Les agitateurs n'étaient nullement coupés du peuple. Ils exprimaient des notions largement propagées parmi les «Hutu» pendant de nombreuses années.

Dès ses premiers numéros, Kangura reformula les thèmes bien connus des «Tutsi » manipulateurs et hypocrites, des femmes «tutsi » ensorceleuses et perfides, tous et toutes s'employant à la conquête du pouvoir. En outre, on trouve dans ces textes l'écho monotone et répétitif d'une rhétorique obsédante sachant manier l'hyperbole :

« Tout Hutu doit savoir que la femme Tutsi où qu'elle soit, travaille à la solde de son ethnie tutsi. Par conséquent, est traître tout Hutu :

- qui épouse une femme Tutsi;

37. MalkKi, 1995, p. 68-73.

38. Dans le présent compte rendu, la petite minorité «Twa », troisième groupe « ethnique », est largement ignorée. Il faut cependant noter que les «Hutu » interrogés par Malkki considéraient les «Twa » à la fois comme moins civilisés et tout aussi «Bantu » qu'euxmêmes. De surcroît, « les Twa étaient idéalisés en tant qu'autochtones ». Sur des fantasmes serbes similaires portant sur les Tsiganes, voir VAN DE PORT, 1994.

39. MaLKKI, 1995, p. 82-88. Il faut savoir qu'au Rwanda, les femmes sont étroitement liées à leur père et à leurs frères. Un autre thème important évoqué par Malkki n'a pas été examiné dans le présent contexte : la présence belge perçue comme force fondamentalement modératrice et bienveillante. Les activistes du Hutu power méprisaient les Belges qu'ils considéraient comme figures de père non fiables, le rôle de soutien paternel étant dévolu aux Français bien qu'on leur reprochât également de ne pas prendre des mesures suffisantes pour protéger leurs « fils préférés », la milice de l'Interahamwe. Voir l'impressionnant recueil de documents de propagande du Hutu power publié in CHRÉTIEN, 1995, p. 278-283. La participation française aux événements est largement examinée in Prunier, 1997, p. 335-369. Voir également KeANE, 1995; et Aucun témoin..., 1999, p. 140-146, 204-206, 763-798. 
— qui fait d'une Tutsi sa concubine;

— qui fait d'une Tutsi sa secrétaire ou sa protégée...

«Tout Hutu doit savoir que tout Tutsi est malhonnête dans les affaires. Il ne vise que la suprématie de son ethnie ${ }^{40}$. »

Le texte présente deux oppositions, l'une majeure entre «Tutsi » et «Hutu », l'autre mineure entre homme et femme. Ces oppositions sont globales et n'admettent aucune exception, comme le martèle la réitération répétitive du mot «tout».

Un nouvel élément intervient : «Les Bahutu doivent cesser d'avoir pitié des Tutsi ${ }^{41}$. » Il s'agit d'une exhortation quasi littérale à la désidentification. Elle suppose cependant qu'une identification préalable a bien eu lieu parmi certains «Hutu» au moins. À ce stade, apparaît la désignation inquiétante des «Tutsi » comme « inyenzi», «cafards ». Les cafards ne cessent de proliférer, ne cessent de manger, et les paysans doivent sans cesse les éliminer ${ }^{42}$. Dès lors, les «Tutsi » sont assimilés à une catégorie générale, abstraite, sans référence à un quelconque événement local ou à un fait historique précis, dissociée de toute relation particulière et entièrement décontextualisée. L'identification des «Tutsi », la désidentification d'avec les «Tutsi » et le refus d'être identifiés avec les «Tutsi », telles sont les conditions nécessaires de la construction identitaire des «Hutu». C'est par projection qu'un ensemble de caractéristiques maléfiques, mais toujours humaines, ont été assignées aux «Tutsi »; c'est par la démesure qu'ils ont été diabolisés jusqu'à leur attribuer un degré de malignité surhumaine; et c'est par la déshumanisation qu'ils ont été ravalés au rang de vermine. Le processus de désidentification est clos. Il quitte même le registre de la haine de l'autre pour atteindre un niveau de froide destruction.

Et pourtant, y compris à ce stade, un processus d'identification complémentaire se poursuit, créant une catégorie « hutu » tout aussi abstraite, soudée par une loyauté réciproque, sans considération de lieu ou de hiérarchie :

«Tout Hutu devrait considérer un autre Hutu comme son frère. Qu'il sache que si demain un des volcans se mettait à cracher, le Hutu du Rukiga serait amené à venir habiter dans le Nduga et à devenir par ce fait même un Munyanduga. Qu'il sache que si une famine survenait au Nduga, un Hutu du Nduga

40. Voir Chrétien, 1995 , p. 39-40, qui cite des extraits de Kangura, n ${ }^{\circ}$ 6, texte publié originellement dans un mélange de kinyarwanda et de français.

41. ChrÉTIEN, 1995, p. 40.

42. Je n'ai pu vérifier si, comme en Europe, les cafards sont également considérés comme « sales ». 
peut aller habiter dans le Rukiga et devenir un Mukiga. Mais, quoi qu'il fasse, un Hutu ne peut devenir un Tutsi et inversement ${ }^{43}$. »

C'était le type de message constamment relayé par le nouveau média, la radio. À la fin des années quatre-vingt, le gouvernement avait distribué un grand nombre de récepteurs dans les milieux ruraux ${ }^{44}$. Comme partout en Afrique, on les transportait avec soi en tous lieux, collés à l'oreille. Ouia ex auditu fides, non ex visu, selon les mots de ce grand propagandiste, Martin Luther : c'est l'écoute qui donne la foi, non la vision.

\section{LE NOUVEAU MODÈLE STANDARD DU CONFLIT ETHNIQUE}

\section{LE CONTEXTE EXPLICATIF}

L'élargissement du champ de l'identification et de la désidentification était une condition nécessaire, mais insuffisante en soi, du génocide à venir. Une démarche explicative approfondie exigerait un compte rendu exhaustif de la constellation économique, politique et militaire, ainsi qu'une analyse précise de la relation entre le mouvement du Hutu power et le régime politique rwandais (comme elle a été publiée récemment par le Human Rights Watch et les ligues des droits de l'homme) ${ }^{45}$. Il serait alors injustifiable de ne pas tenter de faire la distinction entre les craintes réelles d'une invasion militaire réelle, d'une part, et les fantasmes circulant sur la nature diabolique des «Tutsi », d'autre part.

À cette époque, le FPR, l'armée des réfugiés « tutsi » rescapés des massacres de 1959 et des tueries massives qui suivirent, s'était exilée en Ouganda. Ces réfugiés avaient combattu dans les rangs des rebelles victorieux de Museveni. Ils constituaient effectivement une menace militaire réelle aux frontières nord du Rwanda ${ }^{46}$. Parallèlement, dans le Burundi voi-

43. ChrÉtien, 1995, p. 98. Noter l'utilisation de noms de lieux, c'est-à-dire les références à la carte nationale pour la constitution d'une fraternité « hutu » à l'échelle nationale.

44. ChrÉtien, 1995, p. 57. En 1989, on comptait une radio pour treize habitants comparativement à une pour soixante-dix habitants en 1970 .

45. Aucun témoin..., 1999. Sur les relations théoriques entre ces différents niveaux d'analyse pour expliquer le « conflit ethnique », voir KAUfMAN, 1996, p. 149, qui fait une distinction entre : "les explications au niveau d" "image primaire ", centrées sur la nature humaine et le comportement des masses; les explications au niveau d" "image secondaire ", centrées sur la nature des États et le comportement des élites; et les explications au niveau d" image tertiaire ", centrées sur la nature du système international. » Dans le présent article, les sections antérieures traitent essentiellement d'explications au niveau d' «image primaire ».

46. Pour un compte rendu des événements au Rwanda, voir BraecKman, 1994; Keane, 1995; Temmerman, 1994; et, surtout, Aucun témoin..., 1999. Les trois premiers ouvrages cités ont été écrits par des journalistes et sont plutôt favorables aux «Tutsi » victimes d'événements dont ces journalistes furent témoins. Pour un compte rendu plus spécialisé, voir PRUNIER, 1997, et surtout Aucun témoin..., 1999. Depuis les années trente, des prêtres progressistes étaient enclins à compatir avec les «Hutu », opprimés de toujours, et le compte rendu d'un 
sin, la minorité «tutsi »s'était bien évidemment maintenue au pouvoir et continuait de contrôler l'armée. L'oppression de la majorité «hutu » au Burundi se traduisit par des tueries massives qui, en 1972, donnèrent lieu à un «génocide » des «Hutu », tueries qui depuis se sont reproduites régulièrement ${ }^{47}$. De plus, on craignait qu'une fois déclenchée, l'invasion du Nord n'incite les «Tutsi » restés au Rwanda à former une cinquième colonne.

Cependant, la population visée par une campagne de haine à projet génocidaire ne constuait pas nécessairement une menace au sens « réel»: les Juifs en Allemagne n'en représentaient pas une, les Koulaks auraient pu être expropriés plutôt qu'exterminés, et on imagine mal comment les victimes de la Révolution culturelle en Chine auraient pu menacer le régime communiste chinois. En ce sens, l'autonomie relative du fantasme paraît confirmée.

Mais la dialectique de l'identification et de la désidentification joue dans un contexte politique spécifique de désintégration intérieure et de menace extérieure. La dynamique de ce processus peut donc être résumée dans un « nouveau modèle standard de conflit ethnique » que l'on trouve de façon plus ou moins explicite dans la plupart des analyses des conflits issus de la transition postcommuniste, surtout dans l'ex-Yougoslavie : 1) des développements au sein du système transnational des États peuvent éroder le monopole de violence de l'État sur un territoire donné; 2) les acteurs politiques se disputent le privilège d'occuper les postes devenus vacants au sein des institutions de l'État en décomposition; 3) pour asseoir la base de leur pouvoir, ils vont tenter d'utiliser différents dénominateurs de mobilisation politique. Puisant dans la mémoire collective, ils auront recours à toute image commune du passé qui est disponible dans la mémoire collective ; 4) s'ils parviennent à rallier des partisans sous une bannière donnée, ceux qui sont exclus se sentiront menacés, l'État n'étant plus en mesure de les protéger efficacement de la violence; 5) ces exclus se rassembleront [donc] sous une étiquette complémentaire, cherchant un dénominateur organisationnel adéquat dans le réservoir de l'imaginaire historique; 6) à son tour, la formation de ce contre-groupe sera perçue comme une menace par le premier groupe.

Dans les grandes lignes, les événements au Rwanda ressemblent à ceux qui se sont produits en ex-Yougoslavie : au cours d'une période de plus d'un siècle, les transformations sociales avaient produit dans les deux

missionnaire protestant néerlandais transmet implicitement les accents de sa sympathie, voir Overdulve, 1994.

47. Lemarchand, 1996, p. 76 sqq. Lemarchand, 1996, p. 27, considère les massacres de 1972 comme un véritable génocide, à ceci près : il estime qu'il n'existait pas d'intention délibérée d'exterminer la population « hutu». 
régions une transformation des sentiments d'identification à un niveau d'échelle donné qui comportait toutefois, une grande ambivalence. Une crise économique et la menace continue d'invasion avaient érodé l'efficacité de l'état Rwandais. L'effondrement de l'Union soviétique signifiait également la fin de la fédération yougoslave. Les acteurs politiques tentaient de redéfinir les (dés)identifications au sein de la population en maniant d'anciens thèmes dans une version renouvelée. Dans les deux cas, l'État était incapable ou n'était pas disposé à protéger les citoyens qui se trouvaient exclus et menacés. Mais, tandis que l'État yougoslave était effectivement démantelé, l'État croate, et l'État serbe plus encore, étaient assez efficaces dans la protection de ceux qu'ils considéraient comme « leurs » citoyens, et dans la persécution - voire, en Serbie, l'extermination - , de ceux qu'ils avaient exclus de leurs rangs. L'État rwandais, quant à lui, déclencha la campagne génocidaire contre une catégorie de ses citoyens. Au Rwanda comme dans l'ex-Yougoslavie, tout au long des événements, des milices plus ou moins officielles opéraient à côté de la police spéciale et des troupes régulières. Il est certain que ces milices étaient tolérées, même soutenues, par le gouvernement de l'État. Quand cela convenait aux responsables, ils pouvaient toujours nier leur emprise sur ces mêmes milices trop zélées. Mais il est plausible que leurs activités aient, de temps en temps, échappé effectivement au contrôle du gouvernement régulier.

$\mathrm{Au}$ Rwanda, à la veille du génocide, le gouvernement ne pouvait plus contrôler la radicalisation qu'il avait contribué à provoquer ${ }^{48}$. Tandis que le Hutu power était issu du parti gouvernemental, les cercles radicaux s'étaient éloignés d'un régime qui, avec beaucoup d'ambiguïté et d'hypocrisie, avait déjà accepté un accord avec le RPF des exilés tutsi. Le génocide se déclencha avec l'attentat contre le président du Rwanda (et du Burundi), suivi aussitôt par un coup d'État des champions du Hutu power et par l'élimination de tous les éléments modérés au sein du gouvernement. Le génocide rwandais représente donc un cas qui, aussi particulier soit-il, relève plus généralement du modèle standard des conflits ethniques.

Cependant, indépendamment du contexte politique de désintégration du monopole de l'État sur la violence, l'économie rwandaise présentait une grave insuffisance: la terre, ressource essentielle, faisait défaut. $\mathrm{Au}$ Rwanda, la densité de la population atteint trois cents habitants au kilomètre carré. C'est l'un des pays les plus peuplés du monde. Et qui plus est,

48. Les auteurs de Aucun témoin..., 1999, p. 28, rejettent avec force l'idée de « la faillite de l'État » comme un « nouveau cliché », et maintiennent « que l'État rwandais n'avait que trop bien réussi à faire ce que ses dirigeants souhaitaient ». Les auteurs ont, paraît-il, voulu rassembler les faits, en vue d'établir un acte d'accusation, tendant à prouver l'intention génocidaire du gouvernement. Mais ce génocide ne fut pas souhaité par tous ces dirigeants, et ne fut pas non plus uniquement l'œuvre des forces de l'État. 
il n'existe guère d'alternative à l'agriculture. Tout conflit d'intérêts prend une forme particulièrement explosive de jeu à somme nulle: la terre gagnée par l'un est nécessairement perdue par l'autre. Il est donc difficile d'imaginer qu'un compromis et un accord commun entre adversaires puissent jamais profiter à toutes les parties concernées. C'est le fondement matériel qui confère son caractère extrême aux perceptions catégorielles exclusives entre groupes ${ }^{49}$. Si dans des conditions d'anarchie et d'anomie, un groupe se désignant comme «serbe » se forme, mieux vaut chercher refuge auprès d'apparentés «antiserbes » même si, ce faisant, ceux-ci doivent être redéfinis comme musulmans bosniaques ou comme croates bosniaques ${ }^{50}$. Mais le recours à de telles étiquettes ne peut être efficace que s'il s'agit d'éléments, parmi d'autres, déjà inscrits dans un passé construit collectivement $^{51}$.

Dès que la dialectique des identifications et des désidentifications est pleinement à l'œuvre, les fantasmes s'exacerbent les uns les autres. À ce stade, les activistes vont recourir à la violence pour atteindre leur but : évincer les membres du groupe rival des postes de récompense, s'emparer de leur maison, de leur commerce, de leur travail et de leur terre. C'est ça l'aspect d' «action rationnelle » dans la spirale de violence. Le viol constitue une dimension essentielle, symbolique et sexuelle, de cette expropriation. Tous ces actes de violence vont justifier les pires craintes du groupe adverse et l'inciter à réagir de façon identique. Chaque fait sera grossi et fera résonance dans les fantasmes des parties concernées. La présence d'États voisins hostiles et la crainte d'une invasion viendront renforcer et accélérer le processus. Toute intervention étrangère visant à pacifier le conflit entre factions sera perçue comme un acte de guerre par l'une d'entre elles au moins. Les puissances étrangères se verront contraintes à la nonintervention, ou à l'envoi de forces militaires massives pour soumettre l'ensemble de la population. Si aucune puissance étrangère n'est disposée et capable de passer à l'action, seuls l'inertie ou l'arrêt des combats par épuisement mettront un terme à l'affrontement.

Au Rwanda (et au Burundi), chacun possédait des raisons suffisantes de craindre des flambées de violence dès lors que l'État ne pourrait ou ne voudrait plus être garant de la sécurité. Le gouvernement « hutu » au Rwanda était pris au piège entre, d'une part, les milices du Hutu power et, d'autre part, au Nord, la menace de l'armée des anciens réfugiés. Au Burundi, le

49. Je remercie Peter Kloos de m'avoir signalé cet aspect du conflit rwandais.

50. Pour une description du conflit bosniaque considéré sous cet angle, voir SwaAn, 1994.

51. Prunier, 1997, p. 5, oppose clairement les explications «primaire» et « instrumentaliste » d'un conflit ethnique. Mais le point de vue instrumentaliste présuppose que déjà des images collectives de tueries anciennes circulent, images parfois très récentes, sans être toujours factuelles, mais qui sont disponibles comme « instruments primaires » prêts à être utilisés et mobilisés par des entrepreneurs politiques. 
régime «tutsi », sous contrôle quasi total de l'armée, ne pouvait se permettre de renoncer à son monopole de minorité sans s'exposer à être écarté par la majorité « hutu ». Les deux pays vivaient dans la crainte que les événements perpétrés dans l'État voisin ne se reproduisent sur son propre sol, avec l'appui du régime adjacent.

Au cours des années, les cercles d'identification et de désidentification sont passés du cadre du village et de la lignée aux catégories généralisées de «Tutsi» et «Hutu» à l'échelle nationale et même transnationale. Chaque flambée de violence venait alimenter la mémoire collective, fournissant des matériaux bruts aux fantasmes ultérieurs sur la nature du groupe complémentaire. Ainsi se préparait la scène de nouveaux affrontements.

Au Rwanda, la relation entre le mouvement du Hutu power et les milieux gouvernementaux s'est avérée complexe, mais étroite. Même si elle fut parfois désavouée publiquement, la milice reçut l'appui secret ou manifeste - de l'État. En fin de compte, le gouvernement a laissé se perpétrer le génocide et l'armée a pris une part active aux tueries massives. Les massacres, prémédités de longue date, furent l'aboutissement d'une campagne soigneusement orchestrée qui exploita systématiquement les failles de schémas d'identification et de désidentification préexistants et largement répandus, en évolution depuis au moins un siècle.

Abram DE SwAAN*,
Amsterdam $^{\text {se }}$ School voor sociaal wetenschappelijk onderzoek,
Oude Hoogstraat 24,
NL-1012 CE Amsterdam
(octobre 1997-février 2001).

\footnotetext{
* Ce texte, rédigé en anglais en octobre 1997, a été traduit ici en français par Michèle Garlati, avec le soutien financier de l'Académie royale néerlandaise des sciences. Une version préliminaire est parue dans Theory, Culture and Society, vol. XIV, 2 mai 1997, p. 105-122.
} 


\section{LISTE DES RÉFÉRENCES}

Aucun témoin ne doit survivre. Le génocide au Rwanda, 1999, éd., Human Rights Watch. Fédération internationale des ligues des droits de l'homme, Paris, L'Harmattan.

Вӓск (Lucien R.), 1981, « Traditional Rwanda. Deconsecrating a sacred kingdom », in Henri J. M. Claessen et Peter Skalník, éd., The Study of the State, La Haye, Mouton, 1981, p. 15-33.

Bateman (Anthony) et Holmes (Jeremy), 1994, Introduction to psychoanalysis. Contemporary theory and practice, Londres, New York, Routledge.

Bayart (Jean-François), 1989, L'État en Afrique. La politique du ventre, Paris, Fayard.

Bцок (Anton), 1975, Wittgenstein and Elias. Een methodische richtlijn voor de antropologie (Discours d'inauguration), Assen, Van Gorcum.

Braeckman (Colette), 1994, Rwanda. Histoire d'un génocide, Paris, Fayard.

Calhoun (Craig), 1991, «Indirect relationships and imagined communities. Largescale social integration and the transformation of everyday life », in Pierre Bourdieu et James Coleman, éd., Social theory for a changing society, Boulder, CO, Westview Press, p. 95-121.

Chrétien (Jean-Pierre), éd., 1995, Rwanda. Les médias du génocide, Paris, Karthala. Dawkins (Richard), 1986, The Blind Watchmaker, New York, Londres, Norton \& Co.

Elias (Norbert), 1989, Studien über die Deutschen. Machtkämpfe und Habitusentwicklung im 19. und 20. Jahrhundert, éd. Michael SCHRÖTER, Francfort-surle-Main, Suhrkamp.

FLETCHER (Jonathan), 1995, « Towards a theory of decivilizing processes », Amsterdams Sociologisch Tijdschrift, vol. XXII, 2, oct., p. 283-296.

Freud (Sigmund), 1995, Le Malaise dans la culture, Paris, Presses universitaires de France.

Gabbard (Glen O.), 1994, Psychodynamic psychiatry in clinical practice, Washington, American Psychiatric Press.

Gobineau (Joseph), 1933, Essai sur l'inégalité des races humaines, $1^{\text {re }}$ éd. 1853, Paris, Firmin Didot.

Gourevitch (Philip), 1996, «The poisoned country », New York Review of Books, vol. XLIII, 10, 6 juin, p. 58-64.

Hoetink (H.), 1962, De gespleten samenleving in het Caribisch gebied. Bijdrage tot de sociologie der rasrelaties in een gesegmenteerde maatschappij, Assen, Van Gorcum.

KaufMAN (Stuart J.), 1996, «An "international” theory of inter-ethnic war », Review of International Studies, vol. XXII, p. 149-271.

Keane (Fergal), 1995, Season of blood. A Rwandan journey, Harmondsworth, Penguin.

Lame (Danielle R. de), 1996, «Une colline entre mille, ou le calme avant la tempête. Transformations et blocages du Rwanda rural », thèse, Amsterdam, Free University Amsterdam.

Lemarchand (René), 1996, Burundi. Ethnic conflict and genocide, Cambridge, New York/Washington, Cambridge University Press/Woodrow Wilson Center. 
Malkki (Liisa H.), 1995, Purity and exile. Violence, memory, and national cosmology among Hutu refugees in Tanzania, Chicago, Londres, University of Chicago Press.

MalkKi (L. H.), 1996, Réponse à P. Gourevitch, suivie de la réponse de P. Gourevitch, New York Review of Books, vol. XLIII, 14, 19 sept., p. 80-81.

Mennell (Stephen), 1990, «Decivilizing processes. Theoretical significance and some lines of research », International Sociology, vol. V, 2, p. 205-223.

Newbury (Catharine), 1988, The Cohesion of oppression. Clientship and ethnicity in Rwanda, 1860-1960, New York, Columbia University Press.

Overdulve (C. D.), 1994, Rwanda. Volk met een geschiedenis, Kampen, Kok.

Prunier (Gérard), 1997, Rwanda, 1959-1996. Histoire d'un génocide, Paris, Dagorno.

Sagan (Eli), 1985, At the dawn of tyranny. The origins of individualism, political oppression and the State, Londres, Boston, Faber \& Faber.

Shils (Edward), 1991, «Comments » [to Craig Calhoun], in Pierre Bourdieu et James Coleman, éd., Social theory for a changing society, Boulder, CO, Westview Press, p. 126-129.

SwaAn (Abram de), 1994, «State of outrage. The fading line between waging war and fighting crime », European Journal on Criminal Policy and Research, vol. II, 3, p. 7-15.

SwaAn (A. de), 1995, «Widening circles of social identification. Emotional concerns in sociogenetic perspective », Theory, Culture \& Society, vol. XII, 2, p. 25-39.

Temmerman (Els de), 1994, De Doden zijn niet dood. Rwanda, een ooggetuigenverslag, Amsterdam, Anvers, De Arbeiderspers.

Thomas (William I.) et Thomas (Dorothy S.), 1928, The Child in America. Behavior problems and programs, New York, Knopf.

Trouwborst (Albert A.), 1991, «The political economy of the Interlacrustrine States in East Africa », in Henri J. M. Claessen et Pieter VAn de Velde, éd., Early State Economics, New Brunswick, Londres, Transaction (Political and Legal Anthropology Series, vol. VIII), p. 97-108.

VAn De Port (Mattijs), 1994, Het Einde van de wereld. Beschaving. Redeloosheid en Zigeunercafés in Servië, Amsterdam, Babylon-De Geus.

Wesseling (Henk L.), 1991, Verdeel en Heers. De Deling van Afrika, 1889-1914, Amsterdam, Bert Bakker. 